\title{
Tecnologías de la información y la comunicación aplicadas a la educación en ciencias
}

\author{
Danny José Lorduy Flórez ${ }^{1}$, Claudia Patricia Naranjo Zuluaga \\ Universidad de Córdoba, Colombia
}

Autor de correspondencia: ${ }^{1}$ dlorduyflorez@correo.unicordoba. edu.co

Recibido: 06 de junio de 2020 Revisado: 04 de julio de 2020 Aprobado: 21 de septiembre de 2020 Publicado: 24 de octubre de 2020

\section{Resumen}

El uso de las tecnologías de la información y la comunicación [TIC] ha mostrado tener aceptación para aumentar la motivación de los estudiantes, ya que les permite desarrollar competencias tecnológicas, la creatividad y la innovación. El objetivo de la presente investigación fue caracterizar el uso de las TIC en el área de ciencias naturales como factor asociado al aprendizaje de contenidos en ciencia, tecnología y sociedad [CTS]. La muestra estuvo conformada por quince estudiantes de noveno grado y dos docentes del área de ciencias de una institución educativa pública en Colombia. La investigación fue cualitativa con enfoque fenomenológico. Se usaron la observación no participativa, entrevistas semiestructuradas y el análisis de contenido cualitativo. Se observó el cambio en el aprendizaje de los estudiantes con el uso de las TIC en el componente CTS. Este uso propició la motivación, la atención y la participación durante el desarrollo de las actividades. Por lo tanto, es imperativo fortalecer la educación en ciencias desde ambientes tecnológicos para que los estudiantes puedan dar soluciones a situaciones problémicas de su contexto.

Palabras claves: educación en ciencias, ciencia, tecnología y sociedad [CTS], competencias tecnológicas, tecnologías de la información y la comunicación [TIC]

Para citar este artículo: Lorduy, D., \& Naranjo, C. (2020). Tecnologías de la información y la comunicación aplicadas a la educación en ciencias. Praxis \& Saber, 11(27), e11177. https://doi. org/10.19053/22160159.v11.n27.2020.11177 


\title{
Information and communication technologies applied to science education
}

\begin{abstract}
The use of information and communication technologies [ICT] has proven to be accepted to increase students' motivation, as it allows them to develop technological skills, creativity, and innovation. The objective of this research was to characterize the use of ICT in natural sciences as a factor associated with content learning in science, technology, and society [STS]. The sample consisted of 15 ninth-grade students and two teachers from the science area of a public educational institution in Colombia. The research was qualitative with a phenomenological approach. Non-participatory observation, semi-structured interviews, and qualitative content analysis were used. The change in student learning with the use of ICTs was observed in terms of the STS component. This use favoured motivation, attention, and participation during the development of the activities. Therefore, it is imperative to enhance science education from technological environments in order that students can provide solutions to problematic situations in their context.
\end{abstract}

Keywords: science education, science, technology, and society [STS], technological skills, information and communication technologies [ICT]

\section{Tecnologias de informação e comunicação aplicadas ao ensino de ciências}

\section{Resumo}

A utilização das tecnologias de informação e comunicação [TIC] tem demonstrado aceitação para aumentar a motivação dos alunos, permitindo-lhes desenvolver competências tecnológicas, criatividade e inovação. O objetivo desta pesquisa foi caracterizar a utilização das TIC na área das ciências naturais como fator associado à aprendizagem de conteúdos em ciência, tecnologia e sociedade [CTS]. A amostra foi composta por 15 alunos do nono ano e dois professores da área de ciências de uma instituição de ensino pública na Colômbia. A pesquisa foi qualitativa com um enfoque fenomenológico. Foram utilizadas observações não participativas, entrevistas semi-estruturadas e análise qualitativa do conteúdo. A mudança na aprendizagem dos estudantes com o uso das TIC foi observada no componente STS. Este uso incentivou a motivação, a atenção e a participação durante o desenvolvimento das atividades. Portanto, é imperioso fortalecer o ensino de ciências a partir de ambientes tecnológicos para que os alunos possam oferecer soluções para situações-problema em seu contexto.

Palavras-chave: educação em ciência, ciência, tecnologia e sociedade [CTS], competências tecnológicas, tecnologias de informação e comunicação [TIC] 


\section{Introducción}

La educación en ciencias es una tarea ardua que demanda mucha responsabilidad y la labor docente es aceptar ese reto, que implica ayudar al estudiante a construir su propio conocimiento (Caycedo et al., 2016). Asimismo, los procesos de enseñanza y aprendizaje constituyen formas universales del desarrollo psíquico de la persona y son el instrumento esencial de enculturación y humanización (Díaz y Carmona, 2010; Pineda \& Orozco, 2017). Es también el docente el que motiva al estudiante para que trascienda en su proceso educativo sin desistir (Valle et al., 2010). En la actualidad, los estudiantes manejan una relación más cercana con los dispositivos tecnológicos que con los libros y tienden a usar más una sala de informática que una biblioteca (González \& Huerta, 2019).

Es necesario señalar que las tecnologías de la información y la comunicación [TIC] cada día tienen mayor importancia en los diferentes ámbitos de nuestro quehacer laboral, social, y educativo, no solo para procesos administrativos, sino como herramientas fundamentales para apoyar el proceso de enseñanza y aprendizaje (Córdoba et al., 2017). Las TIC en la educación son una herramienta de gran ayuda, puesto que educar con las nuevas tecnologías requiere un conjunto de condiciones didácticas, pedagógicas, económicas, políticas y culturales. No son solo un espacio de información y comunicación, sino también un espacio social (Pérez \& Telleria, 2012). Lo anterior supone una preparación educativa, económica y social que permita a toda la comunidad tener un manejo adecuado de estas herramientas, para así satisfacer realmente las necesidades de estas tecnologías en la educación (Pineda \& Orozco, 2017). Cuando se usan herramientas tecnológicas, suele favorecerse la motivación, la imaginación y la creatividad de los estudiantes. Así se logra un mayor grado de concentración, lo que se ve reflejado en los resultados académicos (Moreno, 2014; Pineda \& Orozco, 2017). Ante este hecho, la motivación actúa como una fuerza intrínseca en los estudiantes, que se compone por el pensamiento, las creencias y las emociones -que surgen y permanecen en ellos-. La motivación está orientada a la realización de las tareas propuestas por el docente (Botero et al., 2017; Paredes \& Arruda, 2012; Torrego-Seijó, 2008).

En las últimas dos décadas, ha aumentado el interés por el estudio de las TIC en investigaciones y en los procesos de enseñanza y aprendizaje de las ciencias naturales y su relación con el componente de ciencia, tecnología y sociedad [CTS] (Chasi \& Lara, 2018; Osorio, 2002; Pineda \& Orozco, 2017). Algunos autores han incluido la letra A de Ambiente a las siglas CTS para dar una imagen más completa y contextualizada de la ciencia, suponiendo considerar la comprensión de cuestiones ambientales y de calidad de vida, sentando las bases para un futuro sostenible (Aguiar-Santos \& Vilches, 2016; Fernandes et al., 2014). Desde el punto de vista del uso de las TIC, las transformaciones en el currículo implican políticas educativas favorables a su uso, el compromiso activo y real de la gestión educativa, la formación del profesorado y una infraestructura básica (Fonseca \& Gamboa, 2017; Jelves, 2017). Asimismo, autores como Cabezas (2016) analizan el origen del componente CTS, al trazar un paralelo entre la tradición europea y la tradición americana. Se establece que la tradición europea pone el énfasis en la dimensión social, mientras que la americana enfatiza en las consecuencias sociales de las innovaciones tecnológicas y su influencia en las formas de vida y en las instituciones. Gordillo (2017) muestra que, al involucrar el componente CTS en educación, se pretende la integración de los individuos con su contexto social, a través de 
la adquisición de los conocimientos, destrezas y actitudes necesarias para su participación en la vida social y el ejercicio de la ciudadanía en sociedades complejas y democráticas.

Por lo tanto, caracterizar el uso de las TIC y su relación con el aprendizaje del componente CTS en el área de ciencias naturales permite que el estudiante haga asociaciones entre lo aprendido y el buen uso de las herramientas tecnológicas, en pro de beneficiar el contexto y la comunidad en general (Córdoba et al., 2017). De igual forma, un enfoque diferente del área de ciencias naturales, al involucrar las TIC, puede favorecer la motivación de los estudiantes y así alcanzar mejores resultados académicos que se vean reflejados en las pruebas practicadas por el Instituto Colombiano para la Evaluación de la Educación, Icfes (Quintero, 2018).

\section{Dificultades en educación en ciencias: una mirada de las TIC y el componente CTS}

Esta investigación está enfocada en las competencias tecnológicas, a la luz de los estándares del Ministerio de Educación Nacional [MEN], las cuales están siendo olvidadas en la educación en ciencias (Maldonado, 2018). Por lo tanto, los estudiantes requieren mayor motivación, dado que se muestran apáticos a algunos procesos de enseñanza que permiten desarrollar competencias tecnológicas. En muchos casos, incluso, tienden a ausentarse de las aulas de clase (Paredes \& Arruda, 2012). Asimismo, el crecimiento exponencial del uso de las TIC y su interacción con el ámbito social y juvenil generan algunas dificultades e incompatibilidades para que los estudiantes se adapten al sistema educativo moderno, debido a los nuevos lenguajes y recursos tecnológicos que predominan (Moreno, 2014). Por lo tanto, es imperativo desarrollar estrategias didácticas y pedagógicas favorables a la atención de los jóvenes, al promover su motivación y restringir los usos de materiales didácticos tradicionales (Moro \& Maris, 2016).

Comúnmente se observa un abandono en los procesos de enseñanza de conceptos relacionados con el componente CTS (Gordillo, 2017). Por lo cual, el docente debe incluirlos en su práctica para propiciar un enfoque por competencias. Sin embargo, deben analizarse los "peligros" que pueden originar los avances tecnológicos en la educación en ciencias (Cárdenas \& Martínez, 2017). En consecuencia, cuando no se tiene en cuenta este componente, es muy probable que se genere desinterés hacia las ciencias en los estudiantes, lo que se evidencia a través de actitudes negativas y predisposición a su aprendizaje (Desuque et al., 2011; Edel \& Ramírez, 2006).

En efecto, la enseñanza de las ciencias debe promover un enfoque por competencias tecnológicas, desde el punto de vista de la aplicabilidad directa de los conocimientos al contexto de los estudiantes (Hinojosa \& Sanmartí, 2016). Con base en lo anterior, la presente investigación gira entorno al siguiente interrogante: ¿qué caracteriza el uso de las TIC en el área de ciencias naturales como factor asociado al aprendizaje de contenidos en CTS?

\section{Metodología}

La indagación en esta investigación fue de tipo cualitativo, con el propósito de describir e interpretar la realidad educativa desde adentro. El diseño fue fenomenológico de vertiente descriptiva, pues se enfoca en obtener las narraciones o descripciones de los docentes y estudiantes. En todo momento se respetan sus propias percepciones acerca del fenómeno 
estudiado (Restrepo, 2018). Este diseño permitió analizar cómo se manifiesta el fenómeno de estudio y sus componentes, recoger información, identificar dificultades y realizar comparaciones en los procesos de enseñanza y aprendizaje de las ciencias (Martínez, 2004; Pérez \& Telleria, 2012). El fenómeno por indagar fue el uso de las TIC en el área de ciencias naturales como factor asociado al aprendizaje del componente de CTS.

\section{Participantes y contexto}

La muestra fue de tipo no probabilístico e intencionada, constituida por un total de quince estudiantes del grado noveno y dos docentes del área de ciencias naturales de la Institución Educativa Ranchería, de la zona urbana del municipio de Sahagún, Córdoba, Colombia. Para este estudio fue necesario utilizar tres técnicas de recolección de datos: la observación no participativa, entrevistas semiestructuradas y la revisión documental.

\section{Análisis de datos}

El tratamiento de los datos se realizó teniendo en cuenta un análisis de contenido cualitativo [ACC] (Krippendorff \& Bock, 2009), por medio de un sistema de categorías, el cual produjo una clasificación semántica entre las categorías y subcategorías de estudio. Para el ACC, se utilizaron seis grabaciones de audio como fuentes de información: 56 minutos en entrevistas semiestructuradas a docentes de ciencias naturales y 83 minutos a estudiantes.

En un primer nivel de análisis, se realizó un proceso de codificación abierta, axial y selectiva con el software Atlas ti. Así, se identificaron presencias, frecuencias y recurrencias en los datos (Strauss \& Corbin, 2002). Después se categorizó la información y se hizo una clasificación semántica para identificar categorías y subcategorías analíticas (Lorduy-Flórez \& Naranjo-Zuluaga, 2020).

\section{Sistema de categorías}

Para definir las categorías y subcategorías de estudio, se analizaron las opiniones de docentes de ciencias [D] y estudiantes [E] de las muestras obtenidas en las entrevistas semiestructuradas, tal como se observa en la tabla 1.

Tabla 1

Sistema de categorías

\begin{tabular}{|c|c|c|c|c|}
\hline $\mathbf{N}^{\mathbf{o}}$ & Objetivos & Categorías & Subcategorías & Preguntas (P) \\
\hline 1 & $\begin{array}{l}\text { Identificar las acciones } \\
\text { de pensamientos del } \\
\text { componente } \\
\text { propuestos en la malla } \\
\text { curricular del grado } \\
\text { noveno, a partir de un } \\
\text { análisis documental de } \\
\text { componentes curriculares } \\
\text { y su relación con los } \\
\text { aprendizajes mediados por } \\
\text { las TIC }\end{array}$ & Currículo & $\begin{array}{l}\text { Plan de } \\
\text { asignatura }\end{array}$ & $\begin{array}{l}\text { E1 ¿Se promueven las competencias } \\
\text { relacionadas con el manejo de las tecnologías } \\
\text { en las ciencias naturales? } \\
\text { E2 ¿Con qué frecuencia realizan experimentos } \\
\text { científicos? } \\
\text { E3 ¿Utilizan los conocimientos aprendidos } \\
\text { para resolver algunos conflictos de la vida } \\
\text { cotidiana? } \\
\text { E4 ¿El docente hace uso de las TIC para el } \\
\text { desarrollo de sus clases de ciencias naturales? } \\
\text { E5 ¿El docente maneja adecuadamente las } \\
\text { herramientas TIC? }\end{array}$ \\
\hline
\end{tabular}




\begin{tabular}{|c|c|c|c|c|}
\hline 2 & $\begin{array}{l}\text { Describir las opiniones } \\
\text { de los docentes respecto } \\
\text { al uso de las TIC y } \\
\text { la enseñanza de los } \\
\text { contenidos de CTS, en el } \\
\text { área de ciencias naturales } \\
\text { en el grado noveno }\end{array}$ & $\begin{array}{l}\text { Prácticas } \\
\text { docentes }\end{array}$ & $\begin{array}{l}\text { Planeación } \\
\text { Uso de las TIC } \\
\text { Trabajo práctico } \\
\text { Evaluación }\end{array}$ & $\begin{array}{l}\text { D1 ¿Hace uso de herramientas TIC para el } \\
\text { desarrollo de sus clases? } \\
\text { D2 ¿Realiza experimentos científicos con } \\
\text { sus estudiantes a fin de mostrar a través de la } \\
\text { práctica lo enseñado? } \\
\text { D3 ¿Promueve la solución de situaciones } \\
\text { de la vida diaria a partir de lo aprendido en } \\
\text { clase? } \\
\text { D4 ¿Promueve el manejo de tecnologías en } \\
\text { clase de ciencias naturales? } \\
\text { D5 ¿Cuál es la respuesta de sus estudiantes } \\
\text { ante el manejo de tecnologías en el desarrollo } \\
\text { de las clases? } \\
\text { D8 ¿Incentiva a sus estudiantes para que } \\
\text { comprueben algunas de las teorías aprendidas } \\
\text { en clase? } \\
\text { D6 ¿Considera usted acertado el número de } \\
\text { evidencias relacionadas con el componente } \\
\text { de CTS? } \\
\text { D9 ¿Realiza actividades que involucren el } \\
\text { desarrollo de la malla curricular con la vida } \\
\text { cotidiana de los estudiantes? } \\
\text { D10 ¿Cómo calificaría la motivación de sus } \\
\text { estudiantes al hacer uso de herramientas } \\
\text { tecnológicas? }\end{array}$ \\
\hline 3 & $\begin{array}{l}\text { Analizar los aprendizajes } \\
\text { de CTS que evidencian } \\
\text { los estudiantes en el grado } \\
\text { noveno desde la enseñanza } \\
\text { de las ciencias naturales y } \\
\text { su relación con el uso de } \\
\text { las TIC }\end{array}$ & $\begin{array}{l}\text { Aprendizajes } \\
\text { de CTS }\end{array}$ & $\begin{array}{l}\begin{array}{l}\text { Diario de } \\
\text { campo }\end{array} \\
\text { Producción } \\
\text { escolar } \\
\text { Aplicabilidad } \\
\text { de la vida diaria }\end{array}$ & $\begin{array}{l}\text { E7 ¿Hace uso de alguna tecnología para } \\
\text { realizar sus exposiciones, tareas o proyectos? } \\
\text { E8 ¿Con qué frecuencia aplica lo aprendido } \\
\text { en clases en su vida diaria? } \\
\text { E9 ¿Le gusta investigar acerca de los } \\
\text { fenómenos que ocurren y de los que se } \\
\text { aprenden a través de la escuela? } \\
\text { E10 ¿Considera importante el uso de las TIC } \\
\text { en su vida diaria? ¿Por qué? } \\
\text { D7 ¿Al realizar las exposiciones o } \\
\text { investigaciones en clases, con qué frecuencia } \\
\text { sus estudiantes hacen uso de herramientas } \\
\text { tecnológicas? }\end{array}$ \\
\hline
\end{tabular}

\section{Fases de la investigación}

Con base en las consideraciones anteriores, se describen las fases del estudio, en donde se detallan las actividades realizadas y el proceso de análisis de los datos obtenidos en la investigación.

\section{Fase 1. Identificación}

A través de esta fase se logró identificar las características del uso de las TIC en el aprendizaje de las ciencias naturales. En ella se señalan las acciones de pensamiento del componente CTS propuestas en la malla curricular del grado noveno y en los Derechos Básicos de Aprendizaje (Gomez \& Velasco, 2017; Hernández-Suárez et al., 2017). Además, se observaron las clases de los docentes de ciencias naturales y se realizaron entrevistas semiestructuradas a docentes y estudiantes con la autorización de los padres de familia, previa a la utilización de herramientas tecnológicas. 


\section{Fase 2. Descripción}

En esta fase se describen las opiniones de los docentes respecto al uso de las TIC y la enseñanza del componente CTS en el área de ciencias naturales en el grado noveno a la luz de la teoría. Para esta fase, se realizó una entrevista semiestructurada a los docentes, la cual fue validada a través del juicio de expertos. Posteriormente, se realizó el respectivo ACC.

\section{Fase 3. Análisis}

En esta fase, se analizaron los aprendizajes adquiridos del componente CTS que evidenciaron los estudiantes y su relación con el uso de las TIC. Además, se analizaron los cuadernos de los estudiantes y se observó la dinámica escolar por medio de ocho visitas a la institución educativa, lo cual se sistematizó mediante un diario de campo.

Lo anterior permitió identificar las temáticas vistas y su relación con el componente CTS, así como la evolución de los aprendizajes y la motivación de los estudiantes después de la utilización de herramientas tecnológicas. Se solicitó y se analizó el plan de clase de los docentes a fin de evidenciar las herramientas tecnológicas que utilizaban para el desarrollo de las temáticas.

\section{Resultados y discusiones}

\section{Acciones de pensamiento}

Después de hacer un análisis documental de la malla curricular para el desarrollo de aprendizajes en ciencias naturales, con base en una matriz de comparación que contiene las acciones de pensamiento propuestas por el MEN, en la cartilla de Estándares básicos de competencias en ciencias naturales (Gómez \& Velasco, 2017), se concluye que solo se trabajan 4 de las 15 acciones de pensamiento propuestas. Se hace énfasis en las que se relacionan con temas de genética, de aplicaciones de microbiología en la industria, del pH dentro del componente del entorno químico y de los avances tecnológicos en comunicaciones y sus implicaciones para la sociedad.

Como una primera aproximación a la caracterización del uso de las TIC desde la categoría currículo, se evidencia que algunos estándares no se cumplen. Sin embargo, los que se registran son de gran utilidad para el desarrollo personal de los estudiantes y la integración de sus conocimientos con el contexto. Lo anterior demuestra una relación directa con el componente CTS, el cual tiene como objeto el estudio de la naturaleza social del conocimiento científico tecnológico y sus incidencias en los diferentes ámbitos económicos, sociales, ambientales y culturales (Osorio, 2002).

\section{Opiniones de los docentes}

Con el fin de describir las opiniones de los docentes respecto al uso de las TIC y la enseñanza de contenidos de CTS en el área de ciencias naturales, se realizaron entrevistas semiestructuradas - fases 1 y 2 - En estas se indagaron temas como: el uso de herramientas tecnológicas en las clases, la motivación del estudiante al hacer uso de las TIC, la aplicación de los contenidos de ciencias naturales en el contexto de los estudiantes, entre otros aspectos.

La figura 1 muestra los resultados relacionados con la categoría prácticas docentes, obtenida de las opiniones de los docentes entrevistados, en relación con el uso de las TIC dentro del aula de clases y la aplicación del componente CTS. Para efectos del análisis de los 
datos, se usan los identificadores $[\mathrm{P}]$ y se indican las preguntas orientadoras de la tabla 1 .

Figura 1

Red semántica: prácticas docentes

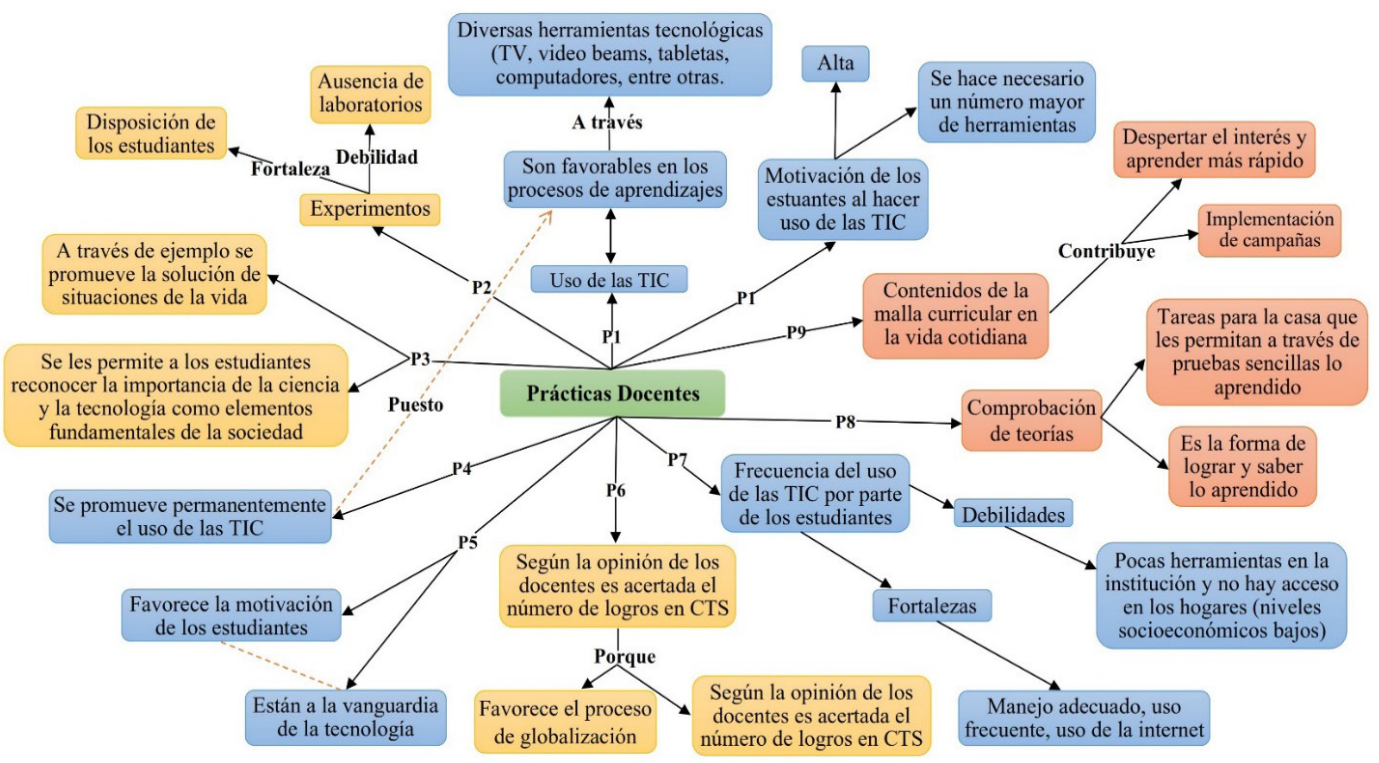

En las relaciones establecidas, se observa la importancia que tiene para los docentes el manejo de las competencias específicas en el componente CTS, tales como: integrar con armonía los temas CTS en el aprendizaje científico - P2 y P8-, hacer ver a los estudiantes la utilidad de la ciencia y la tecnología - P3-, fomentar el uso de las TIC como herramientas de información - P2 y P4-, estudiar las TIC como contenido CTS clave del siglo XXI y educar para la vida y para vivir -P6, P8 y P9- (Vázquez, 2014).

En ese sentido, mediante la elaboración de exposiciones, los docentes fomentan en los estudiantes el aprendizaje con herramientas electrónicas y software - PowerPoint- e información virtual, que les permiten acercarse más al concepto estudiado. Por lo tanto, los ambientes tecnológicos en el proceso educativo permiten la creación de nuevos canales de comunicación y participación, ya que propician competencias tecnológicas que contribuyen permanentemente a la formación del estudiante (Moro \& Maris, 2016). En efecto, la integración de las TIC en las escuelas requiere de actores con las competencias tecnológicas necesarias para efectuar tal emprendimiento (Filippi, 2009). Sin embargo, se refleja poca importancia frente a los avances tecnológicos, así como la resistencia a aceptar dicha expansión en la educación en ciencias (Torres \& Jaimes, 2014).

En relación con el componente CTS, las respuestas de los docentes dejan ver la integración de los conocimientos enseñados con el contexto de los estudiantes al promover el proceso de globalización -P6-. Por tanto, la llegada de la tecnología a las escuelas implica nuevas concepciones del proceso de enseñanza y aprendizaje. El énfasis se traslada desde la enseñanza hacia el aprendizaje con el establecimiento de nuevos roles y responsabilidades para los estudiantes y docentes (Moro \& Maris, 2016). 


\section{Aprendizajes de CTS en los estudiantes}

Con el fin de analizar los aprendizajes en el componente CTS que evidencian los estudiantes desde la enseñanza de las ciencias naturales y su relación con el uso de las TIC, se analizaron los cuadernos de los estudiantes, los planes de clase, la malla curricular, el diario de campo y las respuestas de las entrevistas semiestructuradas - fases 1 y 3 -.

Análisis de los cuadernos de los estudiantes. Después de realizar un análisis en los cuadernos de apuntes de los estudiantes, se observó que gran parte de los temas vistos son abordados por componentes de entorno vivo y entorno físico. Del componente de CTS, solo se logró observar un tema - la microbiología en la industria-. Por lo tanto, el componente de CTS con respecto a los demás es reducido.

Análisis de los planes de clase de los docentes. A partir de la revisión y el análisis de los planes de clases del área de ciencias naturales - fase 3-, se pudo evidenciar que los contenidos vistos durante el año escolar se relacionan principalmente con los componentes de entorno vivo y entorno físico, representados en conceptos de biología, química y física, mientras que los temas vistos en el componente de CTS son reducidos. También se pudo notar que, en la gran mayoría de los planes de clase vistos, son pocas las herramientas tecnológicas utilizadas durante el desarrollo de las clases.

Observación no participante. Análisis del diario de campo. Antes del análisis de la problemática detectada, se realizaron tres visitas iniciales a la institución educativa - fase 3-, en las cuales se analizó que los estudiantes no mostraban mayor motivación en las clases de ciencias naturales. Asimismo, el uso de estrategias didácticas se limitaba al uso de fotocopias e impresiones de libros. Prevalecían las estrategias pedagógicas y didácticas tradicionales y conductistas. En dos ocasiones, varios estudiantes se ausentaron de las clases sin excusa y no volvieron. Mientras se realizó este primer proceso de observación, no se evidenció el uso de las TIC, ni la aplicación del componente CTS por parte de los docentes durante el desarrollo de las clases. Estas tres visitas constituyen una primera experiencia de aproximación y no se observaron cambios en el proceso.

En una cuarta visita se pudo apreciar el uso de computadores, de un televisor y de un videobeam para las exposiciones de los estudiantes. El tema era genética molecular. Las exposiciones estaban divididas en grupos de cuatro estudiantes. Claramente, algunos de ellos no hacían uso de las diapositivas para exponer, sino que el tema era completamente memorizado. Lo anterior permitió contrastar las experiencias de los estudiantes antes y después de la utilización de herramientas tecnológicas durante el desarrollo de las clases.

Este último momento se realizó con la colaboración de seis estudiantes, quienes se encargaron de la instalación de los equipos y de su manipulación en la exposición, lo cual generó una experiencia motivante en ellos, pues hubo un mayor acercamiento a las tecnologías. En las visitas quinta a séptima se repitió lo observado en las tres primeras visitas, salvo que no hubo ausentismo.

En la octava visita, se realizó una tercera experiencia con herramientas tecnológicas: la docente, al finalizar la clase, les solicitó a los estudiantes consultar y analizar, a través de videos de internet o con familiares y conocidos, las siguientes preguntas:

- ¿Cuál es la incidencia de la genética en la vida diaria? 
- ¿Qué rasgos genéticos o hereditarios lo identifican con sus padres y hermanos?

- ¿Cómo afecta la trisomía del par 21 a una persona?

La era tecnológica trae consigo alternativas para mejorar las estrategias de aprendizaje en el aula. Las TIC, como instrumento para la mejora de dichos procesos, han propiciado en los docentes el diseño e implementación de nuevos planes de aula que cumplan con las exigencias que demandan la formación de los estudiantes (Mosalve \& Monsalve, 2015). Sin embargo, la integración de las TIC al proceso enseñanza-aprendizaje de las ciencias no debe pasar por una "moda", oferta promovida por fabricantes tecnológicos o como algo pasajero e intrascendente (Chasi \& Lara, 2018). Se requiere de nuevas miradas que articulen procesos del componente CTS con el uso de las TIC, de tal forma que se promuevan aprendizajes dirigidos hacia la comprensión de los fenómenos sociales, económicos, políticos, ambientales y culturales.

Análisis de las entrevistas a estudiantes. Como resultado de las entrevistas semiestructuradas a los estudiantes en las fases 1 y 3 , antes y después de la utilización de herramientas tecnológicas, se evidenció que, cuando se usaron las TIC, los estudiantes se sintieron más atraídos y motivados hacia el aprendizaje de las ciencias. Sin embargo, se encontraron diversas limitaciones en la aplicación de las estrategias, entre las que están la falta de computadores y de un laboratorio en la institución.

Por otro lado, cuando se implementaron metodologías de enseñanza por medio de las TIC, al final de la fase 3, se observó un aumento en la motivación y atención de los estudiantes. Asimismo, fueron más participativos durante el desarrollo de las actividades, lo que les permitió adentrarse más en su contexto natural. En la figura 2, se muestra el análisis de los datos compilados, obtenidos de las entrevistas a los estudiantes.

En la primera categoría de análisis, que corresponde al currículo, se observa una tendencia a manifestar que las herramientas tecnológicas en las clases de ciencias naturales se utilizan ocasionalmente, por lo que no se observó un desarrollo por competencias tecnológicas. Por lo tanto, en los estudiantes se evidencian tendencias marcadas frente a los perfiles educativos adaptados a la realidad social de su contexto y, por ende, sus modos de aprendizaje son distintos, por lo cual demandan nuevos métodos que se ajusten a sus requerimientos. La integración sinérgica de las TIC propiciaría implícitamente prácticas de adaptación alternativas y modelos de convergencia a los usos habituales de la enseñanza tradicional (Martínez-Argüello et al., 2018).

En cuanto al uso de los conceptos aprendidos, algunos estudiantes en alusión al tema de las ondas dicen:

- El movimiento del agua forma las ondas de las que habla la profesora. (E4)

- A veces, cuando mi mamá grita, hay eco en la sala. (E6)

En relación con la genética expresan:

- La herencia de mi papá son los ojos claros, pero mi color de piel es por mi abuelo que es moreno. Eso quiere decir que me parezco a los dos, por genética. (E3)

Al indagar si el docente hace uso de TIC para el desarrollo de sus clases de ciencias naturales, se encontraron respuestas monosílabas tales como: no -6 veces - y sí -8 veces-. Solo un estudiante expresó: 
- Sí, a ella [docente] le toca en muchas ocasiones traer las cosas de su casa porque en el colegio no alcanzan los que hay, por ejemplo, portátiles, celulares y tabletas. En el colegio hay televisores y videobeam [...] y computadores que a veces funcionan. (E1)

\section{Figura 2}

Red semántica: aprendizajes en CTS

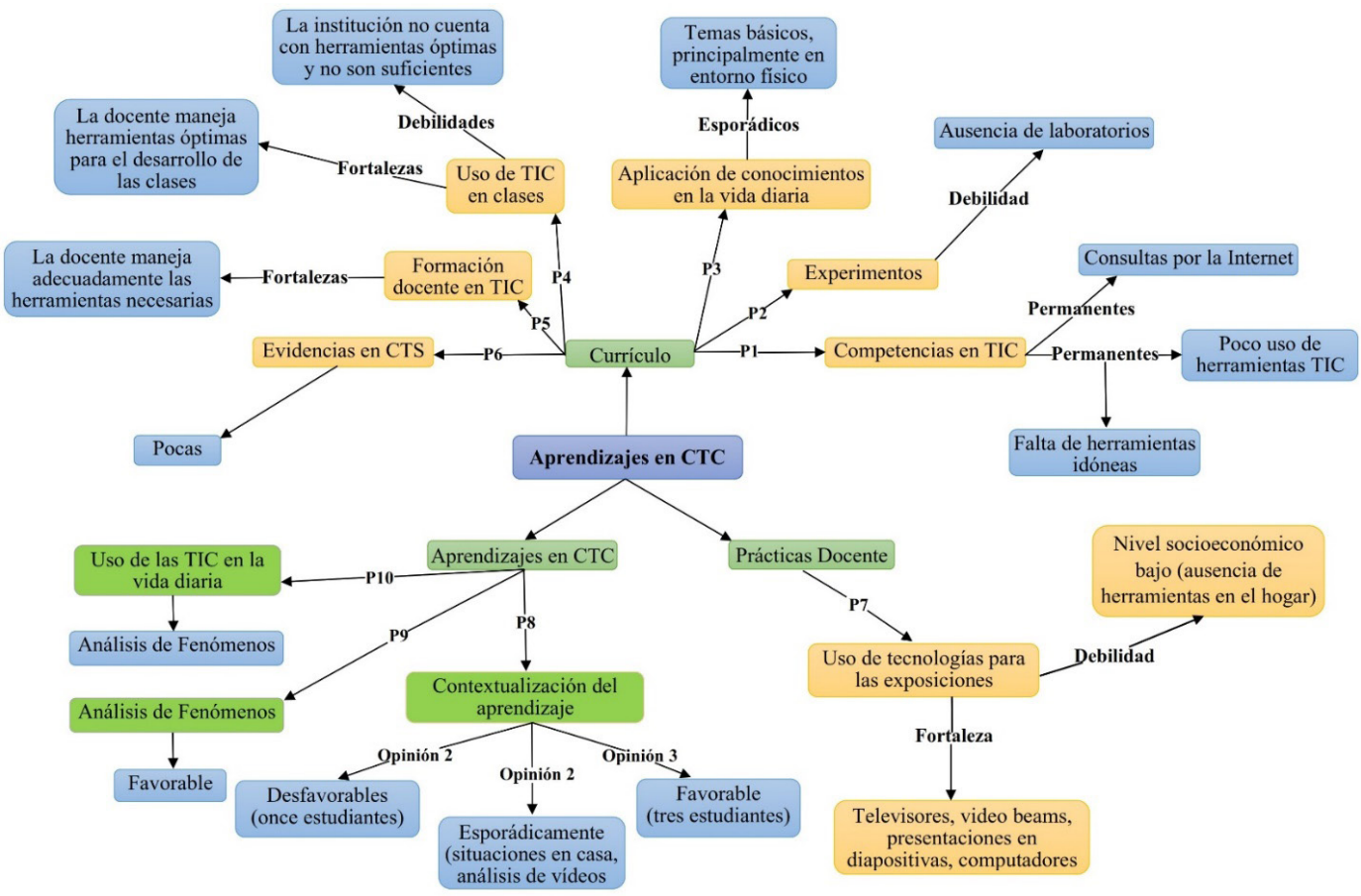

En cuanto a la formación del docente para usar las TIC, todas las respuestas son afirmativas.

- Sí, la profesora conoce bastante bien las herramientas. (E5)

- Sí conoce, pero a veces se ayuda con videos de YouTube cuando no puede solucionar algo de conexión. (E6)

En cuanto a la pregunta acerca de la cantidad de evidencias relacionadas con el componente CTS, ocho de los estudiantes manifiestan estar de acuerdo. Tres de ellos afirman que son pocos y deben ampliarse. Los cuatro restantes solicitaron previa explicación de los contenidos del componente CTS. La categoría prácticas docentes muestra que los estudiantes usan las herramientas tecnológicas para realizar sus exposiciones. Entre estas herramientas mencionan televisores, videobeam, diapositivas, computadores. Salvo tres estudiantes que expresan:

- A mí me toca hacer las exposiciones con cartulina porque no tengo computador. (E8)

- En mi casa no hay computador y a veces Juliana me presta el de ella en su casa y yo hago las diapositivas; cuando no, compramos papel periódico. (E10)

- En la casa no hay computador y no tenemos dinero para pagar un internet. (E4)

En cuanto a la categoría aprendizajes en CTS, la mayoría de los estudiantes manifiestan 
que casi nunca tratan de comprobar si lo aprendido en clases funciona en la vida diaria. Sin embargo, existen excepciones.

- Me gusta buscar en la casa cuando puedo para ver en qué me sirve lo que aprendemos y se puede ver que el sonido produce ondas que crean ecos, y el agua cuando se le arroja una piedra. (E2)

- Busco en la casa o en la calle situaciones que me permitan aplicar lo aprendido. Tiro una piedra al agua y se forman muchas ondas. (E8)

- Sí, con bastante frecuencia trato de ver si lo que aprendí es verdad o me sirve para algo. (E9)

- Busco la forma de relacionar lo que aprendí para saber si en la vida real funciona. (E4)

Se observa también que a los estudiantes les gusta investigar acerca de los fenómenos que ocurren y de los que aprenden a través de la escuela. Un estudiante dice:

- Sí, es interesante buscar acerca de esos fenómenos en internet o leer en libros, o hablar entre los compañeros de ellos, para ver qué es lo que pasa, porque la profesora siempre pone tareas de explicar. (E10)

Además, consideran importante el uso de las TIC en su vida cotidiana y en su contexto social y natural. Algunas de las opiniones son las siguientes:

- Ayudan a estar a la "moda", siempre atentos a los adelantos científicos y tecnológicos, aprendiendo cada día. (E8)

- Ayudan a hacer mejor las actividades escolares, contribuyendo a aprender mejor sobre los problemas que nos rodean. (E6)

Desde el punto de vista de la enseñanza de las ciencias naturales y su relación con el uso de las TIC como factor asociado al componente CTS, se logró observar que no se promueven competencias en cuanto al manejo de tecnologías en las ciencias naturales. En cambio, los estudiantes que manejaron adecuadamente las herramientas tecnológicas mejoraron sus actitudes frente al aprendizaje de las ciencias (Maldonado, 2018). Por el contrario, existe escasa motivación e inconformidad en los estudiantes por la ausencia de un laboratorio para realizar experimentos científicos y de recursos tecnológicos, lo que consideran que sería de gran ayuda en los procesos de aprendizaje. Así se evidencia, una vez más, la necesidad intrínseca de relacionar las TIC con el componente CTS a la hora de aprender ciencias.

Por esta razón, las instituciones educativas deben promover planes de mejora y fortalecimiento para la aplicación del componente CTS desde las TIC, además de favorecer el aprendizaje basado en competencias tecnológicas para poder aplicar los conceptos aprendidos en ciencias naturales. Asimismo, se debe incentivar la transversalidad de las áreas, para que los estudiantes logren apreciar las diversas utilidades de las ciencias naturales en diversos aspectos de la vida diaria desde diferentes puntos de vista.

\section{Conclusiones}

En lo relacionado con la categoría currículo, el análisis de diferentes elementos de la planeación curricular a la luz de las acciones de pensamiento orientadas desde los estándares 
y el componente CTS evidenció que son pocos los contenidos que desarrollan competencias tecnológicas en los estudiantes. En la gran mayoría de los planes de clase analizados, son pocas las herramientas tecnológicas que utilizan los docentes para el desarrollo de sus clases. Asimismo, en el análisis de los cuadernos de los estudiantes, se evidenció un alto contenido en entorno físico y entorno vivo. Del componente CTS, solo se aprecia el tema de microbiología en la industria.

$\mathrm{Al}$ analizar el diario de campo, se puede apreciar que en las clases observadas predomina la enseñanza tradicional y conductista, donde el docente es el eje central en los procesos de enseñanza-aprendizaje. En pocas oportunidades se observó el uso de TIC o el manejo del componente CTS. Los resultados de las entrevistas dejan ver algunas dificultades en el uso de las TIC en el aula, así como su uso fuera de ella.

En cuanto a las opiniones de los docentes respecto al uso de las TIC y la enseñanza de conceptos a través del componente CTS en el área de ciencias naturales en el grado noveno, se logró establecer que para ellos es de suma importancia y gran necesidad hacer uso de estas tecnologías, en la medida que facilitan el aprendizaje de los estudiantes y aumentan su motivación. Sin importar las barreras que se presentan en muchos de los casos - ausencia de laboratorio y materiales tecnológicos-, los docentes buscan alternativas de solución para la implementación de las TIC en el área de ciencias naturales.

En cuanto al componente CTS, los docentes consideran que los cuatro temas establecidos - que constituyeron un hallazgo del primer objetivo - son los que se alcanzan a desarrollar y están relacionados en todas las temáticas como fundamento para el proceso de globalización. Afirman que se ha tratado de enfocar un poco más el conocimiento y desarrollo de contenidos hacia el componente de CTS en las clases. Además, incentivan frecuentemente al estudiante para que ponga en contexto los contenidos aprendidos.

En cuanto al componente CTS, varios de los estudiantes manifestaron curiosidad por aplicar lo aprendido en su vida diaria, a través de asociaciones entre la realidad de su contexto y los temas vistos. Su apreciación en cuanto al manejo de las TIC por parte de los docentes es buena y exaltan su capacidad de reacción ante las carencias de recursos tecnológicos que presenta la institución. También manifiestan que usan algunas tecnologías para realizar sus exposiciones, tareas o proyectos, a través de televisores, videobeam, diapositivas, computadores, salvo por pocos estudiantes que manifiestan no poseer las herramientas necesarias para esto.

Sobre la base de las consideraciones anteriores, los docentes entrevistados usan diversos medios de enseñanza destinados a mejorar los aprendizajes, enfocados en los estudiantes que se encuentran en inclusión educativa y vulnerabilidad social, los cuales están escolarizados en instituciones de educación pública. Si bien, los recursos constituyen un aspecto regular que no contribuye a los anhelos educativos, se resalta el ahínco de los docentes por buscar mejoras en sus metodologías de enseñanza. Sin embargo, hasta el momento, no podemos afirmar que en general se haya producido un cambio en la dinámica educativa debido al uso de las TIC en el área de ciencias naturales. Para ello, estos recursos tendrían que estar asociados al aprendizaje de contenidos en el componente CTS con regularidad en las aulas. 


\section{Referencias}

Aguiar-Santos, D., Vilches, A., \& Brito, L. P. (2016). Importância concedida à CTSA e Sustentabilidade em Revistas de Investigações Científicas Educacionais no Brasil e Espanha. Indagatio Didactica, 8(1), 1808-1820. http://roderic.uv.es/handle/10550/73563

Botero Carvajal, A., Alarcón, D. I., Palomino Angarita, D. M., \& Jiménez Urrego, Á. M. (2017). Pensamiento crítico, metacognición y aspectos motivacionales: una educación de calidad. Poiésis, 1(33), 85. https://doi.org/10.21501/16920945.2499

Cabezas, L. M. (2016). El origen de CTS. Publicaciones Didácticas, 76, 315-320.

Cárdenas, A. M., \& Martínez, C. A. (2017). Los referentes curriculares instituidos para la elaboración del conocimiento escolar en ciencias en Colombia : ¿qué caracteriza la estructura de los estándares básicos de competencias en ciencias? Enseñanza de Las Ciencias: Revista de Investigación y Experiencias Didácticas, Extra, 1183-1188.

Caycedo, L., Trujillo, D., \& García, S. (2016). La responsabilidad social, un componente esencial de la formación en un programa de química ambiental. Misión Jurídica, 9(10), 223-231. https://doi.org/10.25058/1794600x.127

Chasi, E., \& Lara, P. (2018). Capítulo 3: Uso de TIC para dinamizar el proceso de enseñanza aprendizaje. En J. Escobar (Ed.), Tecnología e Innovación + Ciencia e Investigación. CITICI 2018. 42-58. Corporación CIMTED.

Córdoba, M., López, E. E., Ospina, J., \& Polo, J. (2017). Estudiantes de la básica y media con respecto al uso de las TIC como herramientas de apoyo a su aprendizaje. Trilogía Ciencia Tecnología Sociedad, 9(16), 113-125. https://doi.org/10.22430/21457778.178

Desuque, D., Vargas Rubilar, J., \& Lemos, V. (2011). Análisis psicométrico del cuestionario de creencias actitudinales sobre el comportamiento suicida en población adolescente en Entre Ríos, Argentina. Liberabit, 17(2), 187-198.

Díaz, A., \& Carmona Agudelo, N. (2010). La formación integral: una mirada pedagógica desde los docentes. Colombian Applied Linguistics Journal, 29, 7-26.

Edel, R., \& Ramírez, M. (2006). Construyendo el Significado del Cuidado Ambiental: Un Estudio de Caso en Educación Secundaria. REICE: Revista Electrónica Iberoamericana Sobre Calidad, Eficacia y Cambio En Educación, 4(1), 52-70.

Fernandes, I. M., Pires, D., \& Villamañán, R. (2014). Educación científica con enfoque ciencia-tecnología- sociedad-ambiente. construcción de un instrumento de análisis de las directrices curriculares. Formacion Universitaria, 7(5), 23-32. https://doi.org/10.4067/ S0718-50062014000500004

Filippi, J. L. (2009). Método para la integración de Tics: Alicativo a Instituciones Educativas de Nivel Básico y Medio [Tesis de maestría, Universidad Nacional de La Plata]. Repositorio Institucional de la UNLP. https://doi.org/https://doi.org/10.35537/10915/4158

Fonseca, J., \& Gamboa Graus, M. (2017). Aspectos teóricos sobre el diseño curricular y sus particularidades en las ciencias. Boletín Redipe, 6(3), 83-112.

Gomez, P., \& Velasco, C. (2017). Complejidad y coherencia de documentos curriculares 
colombianos: Derechos Básicos de Aprendizaje y Mallas de Aprendizaje. Revista Colombiana de Educación, 73, 261-281. https://doi.org/10.17227/01203916.73rce259.279

González Fernández, M. O., \& Huerta, P. (2019). Experiencia del aula invertida para promover estudiantes prosumidores del nivel superior. RIED. Revista Iberoamericana de Educación a Distancia, 22(2), 245-263. https://doi.org/10.5944/ried.22.2.23065

Gordillo, M. M. (2017). El enfoque CTS en la enseñanza de las ciencias. En S. Duarte (Ed.), Consejo Nacional de Ciencia y Tecnología (CONACYT) - Paraguay, (pp. 24-36).

Hernández-Suárez, C., Pabón Galán, C., \& Prada, R. (2017). Desarrollo de competencias y su relación con el contexto educativo entre docentes de ciencias naturales. Revista Virtual Universidad Católica Del Norte, 51, 194-215.

Hinojosa, J. \& Sanmatí, N. (2016). Indagando en el aula de ciencias: primeros pasos. En J. Sánchez Martín \& F. Cañada (Eds.), Ciencias para comprender el mundo: Investigación e innovación en didáctica de las ciencias experimentales, (pp. 535-542). Entimema.

Jelves Zárate, V. (2017). Develando el Currículo Integrado. Representaciones Sociales que en relación al Currículo Integrado habitan en profesores y profesoras de una escuela que practica la integración curricular. Paulo Freire, 17, 197-223 https://doi. org/10.25074/07195532.17.542

Krippendorff, K., \& Bock, M. A. (2009). The Content Analysis Reader. Sage Publications.

Lorduy-Flórez, D. J., \& Naranjo-Zuluaga, C. P. (2020). Percepciones de maestros y estudiantes sobre el uso del triplete químico en los procesos de enseñanza-aprendizaje. Revista Científica, 3(39), 322-338. https://doi.org/10.14483/23448350.16427

Maldonado, M. (2018). El aula, espacio propicio para el fortalecimiento de competencias ciudadanas y tecnológicas. Sophia, 14(1), 39-50. https://doi.org/10.18634/ sophiaj.14v.1i.822

Martínez-Argüello, L. D., Hinojo-Lucena, F., \& Díaz, I. A. (2018). Aplicación de las Tecnologías de la Información y la Comunicación (TIC) en los Procesos de EnseñanzaAprendizaje por parte de los Profesores de Química. Información Tecnológica, 29(2), 41-52. https://doi.org/10.4067/s0718-07642018000200041

Martínez, M. (2004). Ciencia y arte en la metodología cualitativa. Editorial Trillas.

Mosalve, N., \& Monsalve, C. (2015). La inclusión de la computadora en el aula por docentes de quinto grado de básica primaria como herramienta para propiciar el aprendizaje significativo en los estudiantes. Revista EAN, 79 (50). https://doi.org/10.21158/01208160. n79.2015.1267

Moreno, R. (2014). Las TIC en la enseñanza de las Ciencias Sociales en la Educación Secundaria. Investigación En La Escuela, 0(82), 87-98. https://revistascientificas.us.es/ index.php/IE/article/view/6889

Moro, L., \& Maris, S. (2016). Aprendizaje de ciencias naturales mediado con TIC: estudio de caso de una experiencia innovadora. En Memorias del Seminario Modelos innovadores en las aulas: aprender en la sociedad del conocimiento, escuelas y tecnologías. XVII Encuentro Virtual Educa, San Juan de Puerto Rico 2016. 
Osorio M., C. (2002). La educación científica y tecnológica desde el enfoque en Ciencia, Tecnología y Sociedad: Aproximaciones y experiencias para la educación secundaria. Revista Iberoamericana de Educación, 28(1), 61-81. https://rieoei.org/RIE/article/ view/959

Paredes, J., \& Arruda, R. D. de. (2012). La motivación del uso de las TIC en la formación de profesorado en educación ambiental. Ciência \& Educação (Bauru), 18(2), 353-368. https://doi.org/10.1590/s1516-73132012000200008

Pérez, M., \& Telleria, M. (2012). Las TIC en la educación: nuevos ambientes de aprendizaje para la interacción educativa. Revista de Teoría y Didáctica de Las Ciencias Sociales, 18, $83-112$.

Pineda, E., \& Orozco, P. (2017). Ecosistemas de aprendizaje con gestión de TIC. Una estrategia de formación desde la pedagogía praxeológica. Revista Docencia Universitaria, 17, 71-95.

Quintero Reyes, C. S. (2018). Trabajo de grado práctica profesional en el instituto colombiano para la evaluación de la educación ICFES. In instname:Universidad de Bogotá Jorge Tadeo Lozano. Universidad de Bogotá Jorge Tadeo Lozano. http://hdl. handle.net/20.500.12010/5517

Restrepo, D. (2018). Fenomenología y hermenéutica. Folios, (17), 7.18. https://doi. org/10.17227/01234870.17folios7.18

Strauss, A., \& Corbin, J. (2002). Bases de la investigación cualitativa: técnicas y procedimientos para desarrollar la teoría fundamentada.

Torrego-Seijó, J. (2008). El profesor como gestor del aula. En Hernán, A. y Paredes, J. (Eds.), Didáctica General: La práctica de la enseñanza en Educación Infantil, Primaria y Secundaria (Madrid, Mc.GrawHill), 197-214.

Torres, S. Á., \& Jaimes, K. (2014). Producción de conocimiento mediado Por tic: cuerPos académicos de tres universidades Públicas estatales de méxico. Sinéctica Revista Electrónica de Educación, 44, 2012-2014.

Valle, A., Rodríguez, S., Núñez, J., Cabanach, R., González-Pienda, J., \& Rosario, P. (2010). Motivación y Aprendizaje Autorregulado. Interamerican Journal of Psychology, 44(1), 86-97.

Vázquez, Á. (2014). Enseñanza, Aprendizaje y Evaluación en la Formación de Docentes en Educación CTS en el contexto del siglo XXI. Uni-Pluri/Versidad, 14(2), 37-49. 